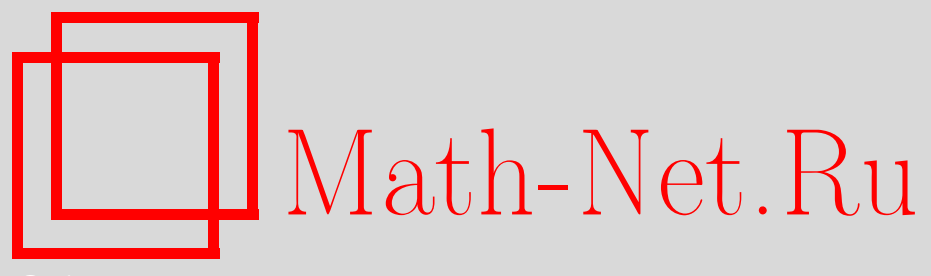

С. Г. Кобельков, О выходах гауссовского процесса с переменной дисперсией за неограниченно растущий барьер, Матем. заметки, 2006, том 80, выпуск 3, 386-394

DOI: https://doi.org/10.4213/mzm2824

Использование Общероссийского математического портала Math-Net.Ru подразумевает, что вы прочитали и согласны с пользовательским соглашением http://www.mathnet.ru/rus/agreement

Параметры загрузки:

IP : 3.80 .253 .173

26 апреля 2023 г., 13:39:35

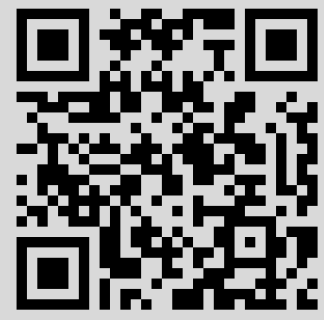


УДК 519.218.7

\section{О ВЫХОДАХ ГАУССОВСКОГО ПРОЦЕССА С ПЕРЕМЕННОЙ ДИСПЕРСИЕЙ ЗА НЕОГРАНИЧЕННО РАСТУЩИЙ БАРЬЕР \\ С. Г. Кобельков}

Для семейства действительнозначных гауссовских процессов $\xi_{u}(t), t \in[0, T]$, при некоторых условиях на дисперсию и корреляцию найдена точная асимптотика вероятности пересечения уровня $u$ при $u \rightarrow \infty$. Данный результат применяется к задаче о выходе стационарного центрированного процесса за неограниченно растущий барьер.

Библиография: 8 названий.

Пусть $\xi_{u}(t), t \in[0, T],-$ семейство действительнозначных гауссовских процессов с нулевым средним и п.н. непрерывными траекториями такое, что дисперсия

$$
\mathrm{E} \xi_{u}(t)^{2}=\sigma_{u}^{2}(t)=1-f(u, t)(1+o(1))
$$

при $u \rightarrow \infty$ равномерно по $t \in[0, T]$, где функция $f(t, u) \geqslant 0$ определена для $u>0$, $0 \leqslant t \leqslant T$ и непрерывна на этом множестве; $f(t, u) \rightarrow 0$ при $u \rightarrow \infty$ равномерно по $t$. Пусть для некоторого $u_{0}>0$ и всех $u>u_{0}$ существует единственное $t_{0}=t_{0}(u)$ такое, что $f\left(t_{0}, u\right)=0, t_{0} \in(0, T)$, и для некоторых $C>0, D_{0}>0, \alpha>0$

$$
\left|f(u, t)-f_{2}(u)\left(t-t_{0}\right)^{2}\right| \leqslant C\left(t-t_{0}\right)^{2+\alpha}, \quad f_{2}(u)>0,
$$

при $|t| \leqslant D_{0}$. Пусть также корреляционная функция $\mathrm{E} \xi_{u}(t) \xi_{u}(s) /\left(\sigma_{u}(t) \sigma_{u}(s)\right)=$ $r_{u}(t, s)<1$ при $t \neq s$ непрерывна, и для любого положительного $\varepsilon$ существуют $\delta(\varepsilon)>0, u_{0}(\varepsilon)>0$ такие, что для всех $u>u_{0},|t-s| \leqslant \delta$ и некоторого $\gamma \in(0,2]$ выполнено

$$
1-\varepsilon \leqslant \frac{1-r_{u}(t, s)}{|t-s|^{\gamma}} \leqslant 1+\varepsilon .
$$

Определим константу Пикандса $H_{\gamma}, 0<H_{\gamma}<\infty$, как [1]

$$
H_{\gamma}=\lim _{T \rightarrow \infty} \frac{H_{\gamma}(T)}{T}, \quad H_{\gamma}(T)=\mathrm{E} \exp \left(\max _{0 \leqslant t \leqslant T}\left(\sqrt{2} B_{\gamma / 2}(t)-t^{\gamma}\right)\right),
$$

где $B_{\gamma / 2}(t)$ - дробное броуновское движение с параметром Харста $\gamma / 2$.

Работа выполнена при финансовой поддержке Российского фонда фундаментальных исследований, грант № 04-01-00700.

(C) С. Г. Коьельков, 2006 
Теорема 1. Пусть выполнены вышеприведенные условия. Тогда

1) если для некоторого $D_{0} \geqslant D_{1}>0$ выполнено $\min _{\left|t-t_{0}\right| \geqslant D_{1}}\left(1-\sigma_{u}^{2}(t)\right) u^{2} \rightarrow \infty$, $u^{2} f_{2}(u) \rightarrow \infty \quad(u \rightarrow \infty), m o$

$$
\mathrm{P}\left(\max _{t \in[0, T]} \xi_{u}(t)>u\right)=\frac{H_{\gamma}}{u^{2-2 / \gamma} \sqrt{f_{2}(u)}} \exp \left\{-\frac{u^{2}}{2}\right\}(1+o(1))
$$

2) если $u^{2} f(u, t) \rightarrow f_{0}(t)$ при $u \rightarrow \infty$ равномерно по $t \in[0, T]$, то

$$
\mathrm{P}\left(\max _{t \in[0, T]} \xi_{u}(t)>u\right)=\frac{H_{\gamma}}{\sqrt{2 \pi} u^{1-2 / \gamma}}\left(\int_{0}^{T} \exp \left\{-f_{0}(t) / 2\right\} d t\right) \exp \left\{-\frac{u^{2}}{2}\right\}(1+o(1)) ;
$$

3) если $u^{2} f(u, t) \rightarrow 0$ при $u \rightarrow \infty$ равномерно по $t \in[0, T]$, то

$$
\mathrm{P}\left(\max _{t \in[0, T]} \xi_{u}(t)>u\right)=\frac{T H_{\gamma}}{\sqrt{2 \pi} u^{1-2 / \gamma}} \exp \left\{-\frac{u^{2}}{2}\right\}(1+o(1)) .
$$

ЗАмЕчАниЕ 1 . В случае 1) достаточно, чтобы корреляция удовлетворяла следующим условиям: $r_{u}(t, s)<1$ при $t \neq s, r_{u}(t, s)$ непрерывна, и для любого положительного $\varepsilon$ существуют $\delta(\varepsilon)>0, u_{0}(\varepsilon)>0$ такие, что для всех $u>u_{0},\left|t-t_{0}\right| \leqslant \delta$, $\left|s-t_{0}\right| \leqslant \delta$ и некоторого $\gamma \in(0,2]$ выполнено

$$
1-\varepsilon \leqslant \frac{1-r_{u}(t, s)}{|t-s|^{\gamma}} \leqslant 1+\varepsilon .
$$

ЗАмЕчАниЕ 2. В случаях 2$), 3)$ достаточно требовать от $f(t, u)$ неотрицательности, непрерывности и соответствующей равномерной сходимости функции $u^{2} f(t, u)$.

СлеДСтвиЕ 1. Пусть $\xi_{u}(t), t \in[0, T],-$ семейство действителънозначных гауссовских процессов с нулевым средним и п.н. непрерывными траекториями. Пусть его дисперсия удовлетворяет соотношению

$$
\mathrm{E} \xi_{u}(t)^{2}=\sigma_{u}^{2}(t)=1-a(u) f(t)(1+o(1))
$$

при $u \rightarrow \infty$ равномерно по $t \in[0, T]$, где функиия $a(u)>0, a(u) \rightarrow 0$ при $u \rightarrow$ $\infty ; f(t)$ - непрерывная функиия такая, что $f\left(t_{0}\right)=0, t_{0} \in(0, T), f(t)>0$ при $t \neq t_{0} u f(t)=f_{2}\left(t-t_{0}\right)^{2}+O\left(\left(t-t_{0}\right)^{2+\alpha}\right), f_{2}>0, \alpha>0$ nрu $t \rightarrow t_{0}$. Пусть также коррелячионная функиия $\mathrm{E} \xi_{u}(t) \xi_{u}(s) /\left(\sigma_{u}(t) \sigma_{u}(s)\right)=r_{u}(t, s)<1$ nри $t \neq$ $s$, непрерывна, $u$ для любого положителъного $\varepsilon$ существуют $\delta(\varepsilon)>0, u_{0}(\varepsilon)>0$ такие, что для всех $u>u_{0},|t-s| \leqslant \delta$ и некоторого $\gamma \in(0,2]$ выполнено

$$
1-\varepsilon \leqslant \frac{1-r_{u}(t, s)}{|t-s|^{\gamma}} \leqslant 1+\varepsilon .
$$

Тогда

1) если $u^{2} a(u) \rightarrow \infty \quad(u \rightarrow \infty)$, то выполнено утверждение 1) теоремы 1 с $f_{2}(u)=a(u) f_{2}$

2) если $u^{2} a(u) \rightarrow c>0$, то выполнено утверждение 2) теоремы 1 c $f_{0}(t)=c f(t)$; 
3) если $u^{2} a(u) \rightarrow 0$, то выполнено утверждение 3) теоремы 1.

ЗАмЕчАниЕ 3. В случае 1) достаточно, чтобы корреляция удовлетворяла следующим условиям: $r_{u}(t, s)<1$ при $t \neq s, r_{u}(t, s)$ непрерывна, и для любого положительного $\varepsilon$ существуют $\delta(\varepsilon)>0, u_{0}(\varepsilon)>0$ такие, что для всех $u>u_{0},\left|t-t_{0}\right| \leqslant \delta$, $\left|s-t_{0}\right| \leqslant \delta$ и некоторого $\gamma \in(0,2]$ будет выполнено

$$
1-\varepsilon \leqslant \frac{1-r_{u}(t, s)}{|t-s|^{\gamma}} \leqslant 1+\varepsilon .
$$

ЗАмЕчаниЕ 4. В случаях 2), 3) теорема верна для непрерывных неотрицательных $f(t)$.

СлЕДствиЕ 2 (задача о движущемся барьере). Пусть $\xi_{t}, t \in[0, T]-$ гауссовский центрированный стационарный процесс с единичной дисперсией, п.н. непрерывными траекториями и ковариационной функцией $R(t), R(t)=1-C t^{\gamma}+o\left(t^{\gamma}\right)$ при $t \rightarrow 0, C>0, \gamma \in(0,2] u|R(t)|<1$ при $t>0$. Функиия $f(t, u)$ определена для $u>0$, $0 \leqslant t \leqslant T$ и непрерьвна на этом множестве. Обозначим $m(u)=\min _{0 \leqslant t \leqslant T} f(t, u)$. Пусть выполнено одно из условий:

1) для каждого и существует единственная точка $t_{0}=t_{0}(u), t_{0} \in(0, T)$, минимума функиии $f(u, t)$ по $t$, причем для некоторых, не зависящих от $t_{0}, D_{0} \geqslant$ $D_{1}>0, C>0, \alpha>0$ выполняются соотношения $\min _{\left|t-t_{0}\right| \geqslant D_{1}} u f(u, t) \rightarrow \infty$, $f(t, u) / u \rightarrow 0$ при $u \rightarrow \infty$ равномерно по $t, u$

$$
\left|\frac{f(u, t)}{u}-\frac{m(u)}{u}-f_{2}(u)\left(t-t_{0}\right)^{2}\right| \leqslant C\left(t-t_{0}\right)^{2+\alpha}, \quad f_{2}(u)>0
$$

$n p u|t| \leqslant D_{0}$, где $u^{2} f_{2}(u) \rightarrow \infty \quad(u \rightarrow \infty) ;$

2) $u f(t, u) \rightarrow f_{0}(t)$ при $u \rightarrow \infty$ равномерно по $t$;

3) $u f(t, u) \rightarrow 0$ при $u \rightarrow \infty$ равномерно по $t$.

Тогда в случае 1$)$ для $P_{f}(u)=\mathrm{P}\left(\max _{0 \leqslant t \leqslant T} \xi_{t}-f(u, t)>u\right)$ справедливо равенство

$$
P_{f}(u)=\frac{H_{\gamma} C^{1 / \gamma}}{(u+m(u))^{2-2 / \gamma} \sqrt{2 f_{2}(u)}} \exp \left\{-\frac{(u+m(u))^{2}}{2}\right\}(1+o(1))
$$

в случае 2)

$$
\begin{aligned}
P_{f}(u)= & \frac{H_{\gamma}}{\sqrt{2 \pi}(u+m(u))^{1-2 / \gamma}}\left(\int_{0}^{T C^{-1 / \gamma}} \exp \left\{-2 f_{0}(t)\right\} d t\right) \\
& \times \exp \left\{-\frac{(u+m(u))^{2}}{2}\right\}(1+o(1)) ;
\end{aligned}
$$

в случае 3 )

$$
P_{f}(u)=\frac{T C^{-1 / \gamma} H_{\gamma}}{\sqrt{2 \pi}(u+m(u))^{1-2 / \gamma}} \exp \left\{-\frac{(u+m(u))^{2}}{2}\right\}(1+o(1)) .
$$


Задача о пересечении уровня, когда среднее и дисперсия исследуемого гауссовского процесса не зависят от уровня, достаточно хорошо изучена. Точная асимптотика соответствующей вероятности в случае стационарного гауссовского процесса была найдена Дж. Пикандсом [2], а нестационарный гауссовский случай был рассмотрен в работе В. И. Питербарга и В. Присяжнюка [3].

Задачей о пересечении движущегося барьера занимались М. Лидбеттер, Дж. Крайер [4], и др. Важной работой является статья С. Бермана [5], где показано, что

$$
\mathrm{P}\left(\max _{0 \leqslant t \leqslant T} X(t)-f(t)>u\right)=F^{\prime}(0)\left(\frac{v}{w}\right)(2 \pi)^{-1 / 2} \frac{e^{-u^{2} / 2}}{u}, \quad u \rightarrow \infty
$$

для стационарного центрированного гауссовского процесса $X(t)$ с единичной дисперсией и ковариационной функцией $r(t)$. Здесь предполагается, что $r(t) \neq 1$ для $t>0$ и правильно меняется в нуле с показателем $2 \geqslant \alpha>0$; кроме того, существует предел $p=\lim _{t \rightarrow 0+} f(t) /(1-r(t))^{1 / 2} ; f(t)$ строго положительна при $t>0$ и правильно меняется в нуле с показателем $\beta \geqslant \alpha / 2 ; v$ и $w$ суть наибольшие решения уравнений $u^{2}(1-r(1 / v))=1, u f(1 / w)=1 . \quad F(x)$ - некоторая функция, которая выражается в явном виде через $\alpha, \beta, p$.

Проблема нахождения вероятностей больших экстремумов гауссовского процесса с переменной дисперсией возникает в задачах аппроксимации [6], [7], задачах о разорении [8] и включает в себя задачу о пересечении движущегося барьера.

Данная работа представляет собой обобщение результатов Бермана, Питербарга и Присяжнюка.

Автор выражает глубокую благодарность профессору В. И. Питербаргу за постановку задачи и полезные обсуждения.

ДокаЗАТЕЛЬСтво теоремы. Пусть $u^{2} f_{2}(u) \rightarrow \infty(u \rightarrow \infty)$. Введем обозначение $\eta(t)=\xi_{u}(t) / \sigma_{u}(t)$. Зададимся произвольным $\varepsilon>0$. Тогда

$$
\begin{aligned}
\mathrm{P}\left(\max _{[0, T]} \eta(t) \sqrt{1-f(u, t)(1+\varepsilon)}>u\right) & \leqslant \mathrm{P}\left(\max _{[0, T]} \xi_{u}(t)>u\right) \\
& \leqslant \mathrm{P}\left(\max _{[0, T]} \eta(t) \sqrt{1-f(u, t)(1-\varepsilon)}>u\right)
\end{aligned}
$$

Возьмем $\beta=\alpha /(4(2+\alpha))$ и положим $\delta=\left(u^{2} f_{2}(u)\right)^{-1 / 2+\beta}$. Обозначим $U=\left[t_{0}-\delta\right.$, $\left.t_{0}+\delta\right], U^{C}=[0, T] \backslash U$. Так как

$$
\begin{aligned}
& \mathrm{P}\left(\max _{t \in U} \eta(t) \sqrt{1-f(u, t)(1 \pm \varepsilon)}>u\right) \leqslant \mathrm{P}\left(\max _{t \in[0, T]} \eta(t) \sqrt{1-f(u, t)(1 \pm \varepsilon)}>u\right) \\
& \leqslant \mathrm{P}\left(\max _{t \in U} \eta(t) \sqrt{1-f(u, t)(1 \pm \varepsilon)}>u\right)+\mathrm{P}\left(\max _{t \in U^{C}} \eta(t) \sqrt{1-f(u, t)(1 \pm \varepsilon)}>u\right)
\end{aligned}
$$


и согласно неравенству Питербарга [1] при $u \rightarrow \infty$

$$
\begin{aligned}
& \mathrm{P}\left(\max _{U^{C}} \eta(t) \sqrt{1-f(u, t)(1 \pm \varepsilon)}>u\right) \\
& \quad \leqslant G_{0} u^{(2 / \gamma)-1} \exp \left\{-u^{2} / 2\right\} \exp \left\{-\frac{u^{2} f_{2}(u) \delta^{2}(1 \pm \varepsilon)(1+o(1))}{2}\right\} \\
& \quad=o\left(u^{(2 / \gamma)-2} f_{2}(u)^{-1 / 2} \exp \left\{-u^{2} / 2\right\}\right),
\end{aligned}
$$

достаточно оценивать вероятности из (1) на $U$.

Возьмем $\Delta=u^{-1} f_{2}(u)^{-1 / 4}$ и разобьем $U$ на отрезки длины $\Delta$. Обозначим через $\Delta_{k} \quad k$-й отрезок разбиения. Пусть $t_{k}^{+}$- точка максимума $f(u, t)$ на $\Delta_{k}$, а $t_{k}^{-}$- точка минимума. Имеем

$$
\begin{aligned}
& \mathrm{P}\left(\max _{t \in U} \eta(t) \sqrt{1-f(u, t)(1-\varepsilon)}>u\right) \\
& \quad \leqslant \sum_{k=1}^{\delta / \Delta} \mathrm{P}\left(\max _{t \in \Delta_{k}} \eta(t)>\frac{u}{\sqrt{1-f\left(u, t_{k}^{+}\right)(1-\varepsilon)}}\right) .
\end{aligned}
$$

По условию для любого $0,5>\varepsilon_{2}>0$ и достаточно больших $u$ на $\Delta_{k}$

$$
1-|t-s|^{\gamma}\left(1-2 \varepsilon_{2}\right)^{\gamma} \leqslant r_{u}(t, s) \leqslant 1-|t-s|^{\gamma}\left(1+2 \varepsilon_{2}\right)^{\gamma}
$$

а следовательно,

$$
\exp \left\{-|t-s|^{\gamma}\left(1-\varepsilon_{2}\right)^{\gamma}\right\} \leqslant r_{u}(t, s) \leqslant \exp \left\{-|t-s|^{\gamma}\left(1+\varepsilon_{2}\right)^{\gamma}\right\}
$$

Обозначим гауссовские центрированные стационарные процессы с ковариационными функциями из $(3) X^{-\varepsilon_{2}}(t)$ и $X^{\varepsilon_{2}}(t)$ соответственно. Тогда согласно лемме Слепяна [1]

$$
\begin{aligned}
\mathrm{P}\left(\max _{t \in \Delta_{k}} X^{\varepsilon_{2}}(t)>\frac{u}{\sqrt{1-f\left(u, t_{k}^{+}\right)(1-\varepsilon)}}\right) & \leqslant \mathrm{P}\left(\max _{t \in \Delta_{k}} \eta(t)>\frac{u}{\sqrt{1-f\left(u, t_{k}^{+}\right)(1-\varepsilon)}}\right) \\
& \leqslant \mathrm{P}\left(\max _{t \in \Delta_{k}} X^{-\varepsilon_{2}}(t)>\frac{u}{\sqrt{1-f\left(u, t_{k}^{+}\right)(1-\varepsilon)}}\right) .
\end{aligned}
$$

Воспользовавшись теоремой D2 [1], получим

$$
\begin{gathered}
\mathrm{P}\left(\max _{t \in \sqrt{1 \pm \varepsilon_{2}} \Delta_{k}} X^{ \pm \varepsilon_{2}}\left(t / \sqrt{1 \pm \varepsilon_{2}}\right)>\frac{u}{\sqrt{1-f\left(u, t_{k}^{+}\right)(1-\varepsilon)}}\right) \\
=\frac{H_{\gamma} \sqrt{1 \pm \varepsilon_{2}}}{u^{1-2 / \gamma} \sqrt{2 \pi}} \Delta \exp \left\{-u^{2} / 2\right\} \exp \left\{-u^{2} f\left(u, t_{k}^{+}\right)(1-\varepsilon) / 2\right\} \\
\quad \times \exp \left\{-\frac{u^{2} f\left(u, t_{k}^{+}\right)^{2}(1-\varepsilon)^{2}}{2\left(1-f\left(u, t_{k}^{+}\right)(1-\varepsilon)\right)}\right\}(1+o(1)) .
\end{gathered}
$$


Заметим, что

$$
\exp \left\{-\frac{u^{2} f_{0}(u)^{2} \delta^{4}(1-\varepsilon)^{2}}{1-f_{0}(u) \delta(1-\varepsilon)}\right\} \leqslant \exp \left\{-\frac{u^{2} f\left(u, t_{k}^{+}\right)^{2}(1-\varepsilon)^{2}}{2\left(1-f\left(u, t_{k}^{+}\right)(1-\varepsilon)\right)}\right\} \leqslant 1 .
$$

и, следовательно, в силу выбора $\delta$

$$
\exp \left\{-\frac{u^{2} f\left(u, t_{k}^{+}\right)^{2}(1-\varepsilon)^{2}}{2\left(1-f\left(u, t_{k}^{+}\right)(1-\varepsilon)\right)}\right\} \rightarrow 1 \quad \text { при } \quad u \rightarrow \infty .
$$

В силу произвольности $\varepsilon_{2}$ из $(4),(5)$ одновременно для всех $k$ имеем

$$
\begin{aligned}
& \mathrm{P}\left(\max _{t \in \Delta_{k}} \eta(t)>\frac{u}{\sqrt{1-f\left(u, t_{k}^{+}\right)(1-\varepsilon)}}\right) \\
& =\frac{H_{\gamma}}{\sqrt{2 \pi} u^{1-2 / \gamma}} \Delta \exp \left\{-\frac{u^{2}}{2}\right\} \exp \left\{-u^{2} f\left(u, t_{k}^{+}\right) \frac{(1-\varepsilon)}{2}\right\}(1+o(1)) .
\end{aligned}
$$

Подставим (6) в $(2)$, воспользовавшись тем, что равномерно по $u$ выполнено $f\left(u, t_{k}^{+}\right)$ $=f_{2}\left(u, t_{k}^{+}\right)^{2}+O\left(\delta^{2+\alpha}\right)$ :

$$
\begin{aligned}
\sum_{k=1}^{\delta / \Delta} \mathrm{P} & \left(\max _{t \in \Delta_{k}} \eta(t)>\frac{u}{\sqrt{1-f\left(u, t_{k}^{+}\right)(1-\varepsilon)}}\right) \\
= & \frac{H_{\gamma}}{u^{1-2 / \gamma} \sqrt{2 \pi u^{2} f_{2}(u)(1-\varepsilon)}} \exp \left\{-\frac{u^{2}}{2}\right\} \sum_{k=1}^{\delta / \Delta} \sqrt{u^{2} f_{2}(u) \Delta^{2}(1-\varepsilon)} \\
& \times \exp \left\{-u^{2} a(u) f_{2}(u)\left(t_{k}^{+}\right)^{2} \frac{(1-\varepsilon)}{2}\right\}(1+o(1)) \\
= & \frac{H_{\gamma}}{u^{1-2 / \gamma} \sqrt{u^{2} f_{2}(u)(1-\varepsilon)}} \exp \left\{-\frac{u^{2}}{2}\right\}(1+o(1)) .
\end{aligned}
$$

Для получения нижней оценки воспользуемся следующим неравенством:

$$
\begin{array}{r}
\mathrm{P}\left(\max _{t \in U} \eta(t) \sqrt{1-f(u, t)(1+\varepsilon)}>u\right) \geqslant \sum_{k=1}^{\delta / \Delta} \mathrm{P}\left(\max _{t \in \Delta_{k}} \eta(t)>\frac{u}{\sqrt{1-f\left(u, t_{k}^{-}\right)(1+\varepsilon)}}\right) \\
\quad-\sum_{i \neq j} \mathrm{P}\left(\max _{t \in \Delta_{i}} \eta(t) \sqrt{1-f(u, t)(1+\varepsilon)}>u, \max _{t \in \Delta_{j}} \eta(t) \sqrt{1-f(u, t)(1+\varepsilon)}>u\right) .
\end{array}
$$

Рассуждениями, аналогичными выводу (7), получим, что

$$
\begin{aligned}
& \sum_{k=1}^{\delta / \Delta} \mathrm{P}\left(\max _{t \in \Delta_{k}} \eta(t)>\frac{u}{\sqrt{1-f\left(u, t_{k}^{-}\right)(1+\varepsilon)}}\right) \\
& \quad=\frac{H_{\gamma}}{u^{1-2 / \gamma} \sqrt{u^{2} f_{2}(u)(1+\varepsilon)}} \exp \left\{-\frac{u^{2}}{2}\right\}(1+o(1)) .
\end{aligned}
$$

Обозначим

$$
P_{i j}=\mathrm{P}\left(\max _{t \in \Delta_{i}} \eta(t) \sqrt{1-f(u, t)(1+\varepsilon)}>u, \max _{t \in \Delta_{j}} \eta(t) \sqrt{1-f(u, t)(1+\varepsilon)}>u\right) .
$$


Представим двойную сумму из (8) в виде

$$
\sum_{i \neq j} P_{i j}=2 \sum_{i+1<j} P_{i j}+2 \sum_{i} P_{i, i+1}=2\left(S_{1}+S_{2}\right) .
$$

Для оценки $S_{1}$ воспользуемся неравенством Питербарга и тем, что для некоторого $\varepsilon_{2}>0$

$$
\max _{t \in \Delta_{i}, s \in \Delta_{j}} \operatorname{Var}(\eta(t)+\eta(s)) \leqslant 4-2 \min \left\{((j-i-1) \Delta)^{2}(1-\varepsilon), \varepsilon_{2}\right\} .
$$

Итак, для $i<j-1$

$$
\begin{aligned}
P_{i j} & \leqslant \mathrm{P}\left(\max _{t \in \Delta_{i}, s \in \Delta_{j}} \eta(t)+\eta(s)>\frac{u}{\sqrt{1-f\left(u, t_{j}^{-}\right)(1+\varepsilon)}}\right) \\
& \leqslant G_{1} u^{2 / \gamma-1} \Delta^{2} \exp \left\{-\frac{u^{2}}{2}\right\} \exp \left\{-u^{2} \Delta^{2}\right\}
\end{aligned}
$$

а в силу выбора $\Delta$ выполнено $\exp \left\{-u^{2} \Delta^{2}\right\} \rightarrow 0$ при $u \rightarrow \infty$. Таким образом, $S_{1}=$ $o\left(u^{(2 / \gamma)-2} f_{2}(u)^{-1 / 2} \exp \left\{-u^{2} / 2\right\}\right)$.

Для оценки $S_{2}$ заметим, что

$$
\begin{aligned}
& P_{i, i+1}= \mathrm{P} \\
&\left(\max _{t \in \Delta_{i}} \eta(t) \sqrt{1-f(u, t)(1+\varepsilon)}>u\right)+\mathrm{P}\left(\max _{t \in \Delta_{i+1}} \eta(t) \sqrt{1-f(u, t)(1+\varepsilon)}>u\right) \\
&-\mathrm{P}\left(\max _{t \in \Delta_{i} \cup \Delta_{i+1}} \eta(t) \sqrt{1-f(u, t)(1+\varepsilon)}>u\right) .
\end{aligned}
$$

Воспользуемся (6) для нахождения асимптотики каждого из слагаемых. Затем, суммируя в (10) аналогично (7), получим

$$
S_{2}=o\left(\frac{H_{\gamma}}{u^{1-2 / \gamma} \sqrt{u^{2} f_{2}(u)(1+\varepsilon)}} \exp \left\{-\frac{u^{2}}{2}\right\}(1+o(1))\right) .
$$

Таким образом, оценка (9) является нижней асимптотической оценкой. В силу произвольности $\varepsilon$ получаем окончательную асимптотику

$$
\mathrm{P}\left(\max _{t \in[0, T]} \xi_{u}(t)>u\right)=\frac{H_{\gamma}}{u^{2-2 / \gamma} \sqrt{f_{2}(u)}} \exp \left\{-\frac{u^{2}}{2}\right\}(1+o(1)) .
$$

В случае, когда $u^{2} f(t, u) \rightarrow f_{0}(t)$ при $u \rightarrow \infty$ равномерно по $t$, положим $\delta=T$, $\Delta=u^{-1 / 2}, U=[0, T]$. Тогда остаются справедливыми (1), (2), (6), а (7) и (9) перепишутся в виде

$$
\begin{aligned}
& \sum_{k=1}^{\delta / \Delta} \mathrm{P}\left(\max _{t \in \Delta_{k}} \eta(t)>\frac{u}{\sqrt{1-f\left(u, t_{k}^{ \pm}\right)(1 \mp \varepsilon)}}\right) \\
& \quad=\frac{H_{\gamma}}{u^{1-2 / \gamma} \sqrt{2 \pi}} \exp \left\{-\frac{u^{2}}{2}\right\} \sum_{k=1}^{\delta / \Delta} \Delta \exp \left\{-u^{2} f\left(u, t_{k}^{ \pm}\right) \frac{(1 \mp \varepsilon)}{2}\right\}(1+o(1)) \\
& \quad=\frac{H_{\gamma}}{u^{1-2 / \gamma} \sqrt{2 \pi}} \exp \left\{-\frac{u^{2}}{2}\right\}\left(\int_{0}^{T} \exp \left\{-f_{0}(t) \frac{(1 \mp \varepsilon)}{2}\right\} d t\right)(1+o(1))
\end{aligned}
$$


Отсюда в силу произвольности $\varepsilon$ легко следует требуемая верхняя оценка:

$$
\mathrm{P}\left(\max _{t \in[0, T]} \xi_{u}(t)>u\right) \leqslant \frac{H_{\gamma}}{u^{1-2 / \gamma} \sqrt{2 \pi}}\left(\int_{0}^{T} \exp \left\{-\frac{f_{0}(t)}{2}\right\} d t\right) \exp \left\{-\frac{u^{2}}{2}\right\}(1+o(1)) .
$$

Доказательство того, что нижняя оценка совпадает с верхней, повторяет соответствующие рассуждения для случая 1).

Если $u^{2} f(t, u) \rightarrow 0$ равномерно по $t$, то для $\delta=T, \Delta=u^{-1 / 2}, U=[0, T]$ также остаются справедливыми (1), (2), (6), а (7) и (9) преобразуются в

$$
\begin{aligned}
& \sum_{k=1}^{\delta / \Delta} \mathrm{P}\left(\max _{t \in \Delta_{k}} \eta(t)>\frac{u}{\sqrt{1-a(u) f\left(t_{k}^{ \pm}\right)(1 \mp \varepsilon)}}\right) \\
& \quad=\frac{T H_{\gamma}}{u^{1-2 / \gamma} \sqrt{2 \pi(1 \mp \varepsilon)}} \exp \left\{-\frac{u^{2}}{2}\right\}(1+o(1)) .
\end{aligned}
$$

Проводя рассуждения, аналогичные доказательству теоремы для случая 1), получим справедливость теоремы для случая 3). Теорема доказана.

ДокАЗАТЕЛЬСТво СЛЕДСТВИЯ 2. Обозначим $D=C^{-1 / \gamma}$.

Рассмотрим случай $m(u)=0$. Тогда $f(u, t) \geqslant 0$. Очевидно, выполнено равенство

$$
\mathrm{P}\left(\max _{0 \leqslant t \leqslant T} \xi_{t}-f(u, t)>u\right)=\mathrm{P}\left(\max _{0 \leqslant t \leqslant T D^{-1}} \frac{u}{u+f(u, D t)} \xi_{D t}>u\right) .
$$

Дисперсия процесса $\frac{u}{u+f(u, D t)} \xi_{D t}$ равна

$$
\sigma_{u}(t)^{2}=1-2 \frac{f(u, D t)}{u}+O\left(\frac{f(u, D t)}{u^{2}}\right) .
$$

Для корреляционной функции $r_{u}(t, s)=R(D(t-s))$ выполнены условия теоремы 1. Таким образом, в случае 1)

$$
\begin{aligned}
& \mathrm{P}\left(\max _{0 \leqslant t \leqslant T} \xi_{t}-f(u, t)>u\right) \\
& \quad=\frac{H_{\gamma}}{\sqrt{2 \pi} u^{1-2 / \gamma}}\left(\int_{0}^{T C^{-1 / \gamma}} \exp \left\{-2 f_{0}(t)\right\} d t\right) \exp \left\{-\frac{u^{2}}{2}\right\}(1+o(1))
\end{aligned}
$$

в случае 2)

$$
\mathrm{P}\left(\max _{0 \leqslant t \leqslant T} \xi_{t}-f(u, t)>u\right)=\frac{T C^{-1 / \gamma} H_{\gamma}}{\sqrt{2 \pi} u^{1-2 / \gamma}} \exp \left\{-\frac{u^{2}}{2}\right\}(1+o(1))
$$

в случае 3)

$$
\mathrm{P}\left(\max _{0 \leqslant t \leqslant T} \xi_{t}-f(u, t)>u\right)=\frac{H_{\gamma} C^{1 / \gamma}}{u^{2-2 / \gamma} \sqrt{2 f_{2}(u)}} \exp \left\{-\frac{u^{2}}{2}\right\}(1+o(1)) .
$$

Откажемся от предположения о равенстве нулю $m(u)$. Тогда

$$
\mathrm{P}\left(\max _{0 \leqslant t \leqslant T} \xi_{t}-f(u, t)>u\right)=\mathrm{P}\left(\max _{0 \leqslant t \leqslant T} \xi_{t}-(f(u, t)-m(u))>u+m(u)\right) .
$$

Так как $u+m(u) \rightarrow \infty$ при $u \rightarrow \infty$, задача сводится к уже рассмотренному случаю. 


\section{СПИСОК ЦИТИРОВАННОЙ ЛИТЕРАТУРЫ}

[1] В.И. Питербарг, Асимптотические методы в теории гауссовских случайных прои,ессов и полей, Изд-во МГУ, М., 1988.

[2] J. Pickands, III, "Upcrossing probabilities for stationary Gaussian processes", Trans. Amer. Math. Soc., 145 (1969), 51-73.

[3] В. И. Питербарг, В. Присяжнюк, "Асимптотическое поведение вероятности большого выброса для нестационарного гауссовского процесса", Теория вероятн. и матем. статистика, 18 (1978), 121-134.

[4] M. R. Leadbetter, "On crossings of arbitrary curves by certain Gaussian processes", Proc. Amer. Math. Soc., 16 (1965), 60-68.

[5] С. Берман, "Выбросы стационарного гауссовского процесса за высокий движущийся барьер", Случайные прочессы. Выборочные функиии и пересечения, Мир, М., 1978, 133-164.

[6] V. Piterbarg, O. Seleznjev, Linear interpolation of random processes and extremes of a sequence of Gaussian non-stationary processes, Proc. Tech. Rep., 446, North Carolina Univ., Chapel Hill, Center Stoch., 1994.

[7] J. Hüsler, V. Piterbarg, O. Seleznev, "On convergence of the uniform norms for Gaussian processes and linear approximation problems", Ann. Appl. Probab., 13:4 (2003), 16151653.

[8] E. Hashorva, J. Husler, "Extremes of Gaussian processes with maximal variance near the boundary points", Methodol. Comput. Appl. Probab., 2:3 (2000), 255-269.

\section{С. Г. Кобельков}

Поступило

Московский государственный университет

14.01.2005

им. М. В. Ломоносова 